\title{
The impact of the Tracey judgment on the rates and outcomes of in-hospital cardiac arrests in UK hospitals participating in the National Cardiac Arrest Audit s s $^{\prime \prime \prime}$
}

\author{
Authors: Zohra Zenasni, ${ }^{\mathrm{A}}$ Emily C Reynolds, ${ }^{\mathrm{B}}$ David A Harrison, ${ }^{\mathrm{C}}$ Kathryn M Rowan, ${ }^{\mathrm{D}}$ Jerry P Nolan, ${ }^{\mathrm{E}}$ \\ Jasmeet Soar ${ }^{F}$ and Gary B Smith ${ }^{G}$
}

\begin{abstract}
Aims
The aim was to determine if the 17 June 2014 Tracey judgment regarding 'do not attempt cardiopulmonary resuscitation' decisions led to increases in the rate of in-hospital cardiac arrests resulting in a resuscitation attempt (IHCA) and/or proportion of resuscitation attempts deemed futile.

Method

Using UK National Cardiac Arrest Audit data, the IHCA rate and proportion of resuscitation attempts deemed futile were compared for two periods (pre-judgment (01 July 2012 - 16 June 2014, inclusive) and post-judgment (01 July 2014 - 30 June 2016, inclusive)) using interrupted time series analyses.

Results

A total of 43,109 IHCAs (115 hospitals) were analysed. There were fewer IHCAs post- than pre-judgment (21,324 vs 21,785, respectively). The IHCA rate was declining over time before the judgment but there was an abrupt and statistically significant increase in the period immediately following the judgment $(p<0.001)$. This was not sustained post-judgment. The proportion of resuscitation attempts deemed futile was smaller post-judgment than pre-judgment $(8.2 \%$ vs $14.9 \%$, respectively). The rate of attempts deemed futile decreased post-judgment $(p<0.001)$.

Conclusion

The IHCA rate increased immediately after the Tracey judgment while the proportion of resuscitation attempts deemed
\end{abstract}

Authors: ${ }^{\text {A }}$ statistician, Intensive Care National Audit \& Research Centre, London, UK; ${ }^{B}$ registrar in intensive care medicine, Liverpool Hospital, Sydney, Australia; ${ }^{C}$ head statistician, Intensive Care National Audit \& Research Centre, London, UK; D professor and director of scientific and strategic development, Intensive Care National Audit \& Research Centre, London, UK; ${ }^{\text {Eprofessor of }}$ resuscitation medicine, University of Warwick, Warwick, UK and consultant in anaesthesia and intensive care medicine, Royal United Hospital, Bath, UK; ${ }^{F}$ consultant in anaesthesia and intensive care medicine, Southmead Hospital, Bristol, UK; ' visiting professor, Bournemouth University, Bournemouth, UK; on behalf of the National Cardiac Arrest Audit futile decreased. The precise mechanisms for these changes are unclear.

KEYWORDS: Resuscitation, cardiac arrest, Tracey judgment, DNACPR, do not attempt cardiopulmonary resuscitation

DOI: 10.7861/clinmed.2019-0454

\section{Background}

On 17 June 2014, the Court of Appeal for England and Wales ruled that the human rights of Mrs Janet Tracey were breached when a do not attempt cardiopulmonary resuscitation (DNACPR) notice was placed in her clinical notes without her knowledge. ${ }^{1}$ Mrs Tracey was 63 years old and was diagnosed with terminal lung cancer in February 2011. Two weeks later, she sustained a high cervical spine injury in a road accident. She required mechanical ventilation because of chronic respiratory disease. After two unsuccessful attempts to wean her from ventilatory support, a DNACPR decision was made by the clinical team. According to the court judgment, the evidence indicated that this occurred without discussion with her or her family, despite evidence that Mrs Tracey wished to be involved in decisions about her medical care. ${ }^{1}$ After Mrs Tracey was successfully weaned from mechanical ventilation, her daughter learned of the DNACPR decision and objected on the basis that there had been no consultation. In response, the DNACPR decision was rescinded. A few days later, Mrs Tracey's condition deteriorated and, following discussions with her family, a new DNACPR decision was made. ${ }^{1}$ Mrs Tracey died on 07 March 2011.

At the 2014 judicial review, Lord Dyson stated that 'since a DNACPR decision is one which will potentially deprive the patient of life-saving treatment, there should be a presumption in favour of patient involvement. There need to be convincing reasons not to involve the patient'. This remains the case even where a DNACPR decision is made because there is no realistic chance that cardiopulmonary resuscitation (CPR) would be successful. The judgment also clarified that a patient is entitled to a second opinion where they disagree with a DNACPR decision. However, it confirmed that it is inappropriate to involve the patient if the 
clinician considers that to do so is likely to cause the person "to suffer physical or psychological harm.1

A systematic review of research published prior to the judgment showed that the involvement of patients in DNACPR discussions was variable, ranging from $25 \%$ to $82 \%$, despite evidence that patients wish to discuss resuscitation. ${ }^{2-4}$ Underlying reasons for staff not involving patients included a desire to avoid causing anxiety or distress to the patient; staff feeling inadequately trained, experienced, or confident; and the fear of complaints. ${ }^{5-11}$

We hypothesised that the judgment, combined with a reluctance by staff to discuss DNACPR recommendations with patients and their families, would lead to a reduction in DNACPR decisions. We considered that this would lead to an increase in the rate of inhospital cardiac arrests resulting in a resuscitation attempt (IHCA; measured as IHCAs per 1,000 hospital admissions), as patients for whom CPR would have no realistic chance of success would receive it because they had no recorded DNACPR recommendation. We hypothesised that this would also lead to an increase in the proportion of resuscitation attempts terminated early because continuation was deemed futile. Therefore, we inspected the National Cardiac Arrest Audit (NCAA) database and compared data from before and after the judgment.

\section{Methods}

\section{National Cardiac Arrest Audit}

NCAA is the UK national clinical audit of patients greater than 28 days of age in acute hospitals in the UK who receive CPR following an in-hospital cardiac arrest and are attended by the hospitalbased resuscitation team (or equivalent) in response to a 2222 call (2222 is the emergency telephone number used to summon a resuscitation team in UK hospitals). CPR is defined by NCAA as the receipt of chest compressions and/or defibrillation.

NCAA collects data according to a standardised dataset. ${ }^{12}$ These include patient identifiers (eg age, sex and ethnicity), and information about the patient's admission (eg date and reason), IHCA (eg date/time of 2222 call, location, presenting/ first documented rhythm and reason resuscitation stopped) and outcome (eg post-IHCA location, status at discharge from hospital and date of hospital discharge/death). Data are entered onto a secure online system and validated both at the point of entry and centrally. NCAA has approval from the Confidentiality Advisory Group within the Health Research Authority to hold patient identifiable data under Section 251 of the NHS Act 2006 (approval number ECC 2-06(n)/2009).

\section{Inclusion and exclusion criteria}

All resuscitation team visits for IHCAs in non-specialist hospitals participating in NCAA from 01 July 2012 to 30 June 2016 were included in the initial dataset. For inclusion, hospitals had to have contributed data from at least 01 July 2013 until 30 June 2015. Five cardiothoracic/chest hospitals and four paediatric hospitals were excluded from the initial dataset due to their very different patient casemix. The following IHCAs were excluded: those involving patients under 16 years, staff, hospital visitors or outpatients; and those where data regarding the reason for stopping CPR were not available. Prehospital cardiac arrests were also excluded.

\section{Data}

The following data were extracted for all included IHCAs: age; sex; ethnicity; reason for admission to hospital; hospital length of stay prior to IHCA; day/time of 2222 call; location of IHCA; presenting/ first documented IHCA rhythm; reason resuscitation was stopped and post-IHCA location.

\section{Primary and secondary objectives}

The primary objectives of the study were to determine whether, following the judgment, there was an increase in the rate of IHCAs and the proportion of resuscitation attempts deemed futile, defined by NCAA as 'resuscitation stopped early as continuation deemed futile when the team arrives'. Data from two periods were compared, pre-judgment (01 July 2012 - 16 June 2014, inclusive; 716 days) and post-judgment (01 July 2014 - 30 June 2016, inclusive; 731 days). Data from 17 June 2014 - 30 June 2014, inclusive (14 days), the period immediately after the judgment was delivered, were excluded from analyses. This was an ad hoc decision, arbitrarily chosen to ensure time for the judgment to become widely available.

\section{Analyses}

A statistical analysis plan was agreed a priori. Descriptive statistics were used to compare the following factors in the pre- and postjudgment periods: number of IHCAs; number of patients; age; sex; ethnicity; reason for admission to hospital; hospital length of stay prior to IHCA; day/time of 2222 call; location of IHCA; presenting/ first documented IHCA rhythm; reason resuscitation was stopped and post-IHCA location. Trends in the reasons for resuscitation being stopped were plotted by month using line graphs.

The total IHCA rates were analysed with an interrupted time series analysis using multilevel Poisson regression (with random effects of hospital), with a primary exposure of the post-judgment compared to pre-judgment period, and a change in time trend. ${ }^{13}$ These analyses were adjusted for seasonality (month of the year). Similarly, the proportion of resuscitation attempts deemed futile was analysed with an interrupted time series analysis using multilevel logistic regression with a primary exposure of the postjudgment compared with pre-judgment period and change in time trend, adjusted for the variables in the NCAA risk model. ${ }^{13,14}$

The analyses were repeated for two IHCA subgroups: those occurring in a ward and those where the patient had a hospital length of stay of $>24$ hours before the IHCA. Ad hoc analyses were also undertaken for the proportion of medical admissions aged $>70$ years who remained on a ward rather than being admitted to the intensive care unit (ICU) following a successful resuscitation attempt - this being a possible surrogate for care limitation.

All analyses were performed using Stata/SE V14.2 (StataCorp, Texas, USA).

\section{Results}

Between 01 July 2012 and 30 June 2016, NCAA recorded a total of 52,341 team visits for IHCAs in non-specialist hospitals. After exclusions (see supplementary material S1), a total of 43,109 IHCAs from 115 adult, acute hospitals remained for analysis (prejudgment, 21,785 in 20,922 individuals; post-judgment, 21,324 in 20,517 individuals). 


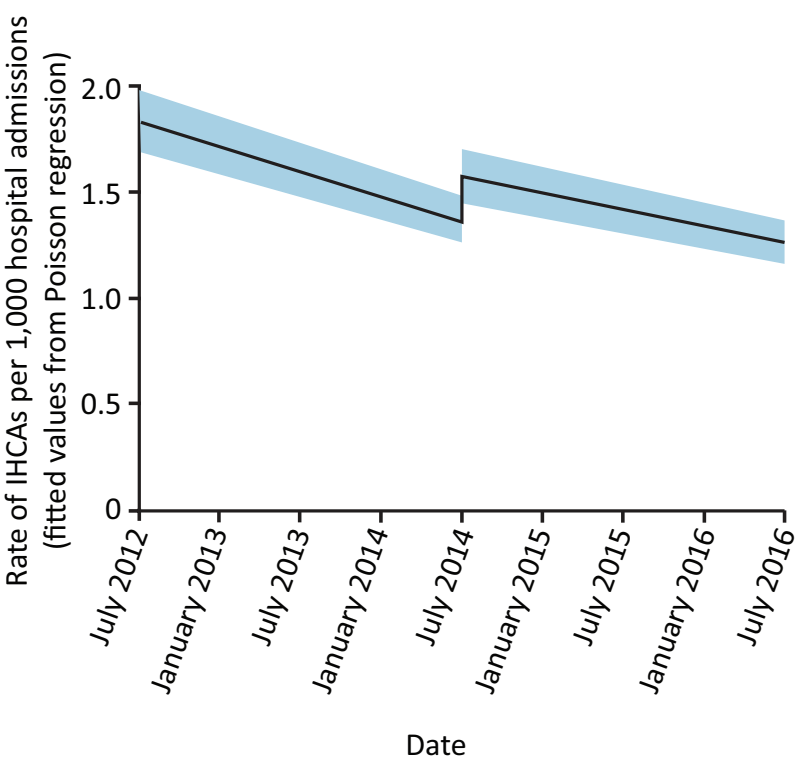

Fig 1. Interrupted time series analysis showing the impact of the Tracey judgment (published on 17 June 2014) on the rate of IHCAs per 1,000 hospital admissions. IHCAs $=$ in-hospital cardiac arrests resulting in a resuscitation attempt.

Descriptive statistics for the pre- and post-judgment periods are shown in supplementary material S2. Patient characteristics (age, sex and ethnicity) were similar in the two groups. The majority of patients were medical admissions (pre-judgment $82.6 \%$; post-judgment $85.0 \%$ ) and most IHCAs occurred on wards (pre-judgment $68.9 \%$; post-judgment $68.4 \%$ ).

Fig 1 shows that the IHCA rate was declining over time before the judgment (incidence rate ratio (IRR) 0.87 per year; $95 \%$ confidence interval (CI) 0.84-0.89), but that a sudden, abrupt and statistically significant increase in the IHCA rate occurred immediately following the judgment delivery (IRR 1.14; 95\% CI 1.10-1.19; $p<0.001$ ). After the judgment, the earlier gradual decline in IHCA rate resumed, but at a marginally slower rate (post-judgment trend IRR 0.90 per year; 95\% CI 0.87-0.92; $\mathrm{p}=0.036$ for pre- vs post-judgment trend).

Fig 2 shows the pattern over time of the reasons why resuscitation was stopped. Over the study period, the proportions of IHCAs with no return of spontaneous circulation (ROSC) and those with ROSC $>20$ minutes (ie alive) increased from $45.0 \%$ to $48.1 \%$ and from $30.0 \%$ to $35.1 \%$, respectively. The proportions of IHCA where a documented valid DNACPR notice was discovered after CPR had commenced and those where spontaneous circulation was sustained for less than 20 minutes (ie ROSC $<20$ min) decreased from $2.9 \%$ to $2.3 \%$ and from $7.3 \%$ to $6.3 \%$, respectively. The proportion of resuscitation attempts deemed futile decreased from $14.9 \%$ to $8.2 \%$. The logistic regression model for the proportion of resuscitation attempts deemed futile indicated no step change in the proportion following the judgment (odds ratio (OR) 1.00; $95 \%$ CI $0.88-1.13 ; p=0.96$ ) but a statistically significant steepening of the rate at which futile resuscitation attempts were decreasing over time; pre-judgment trend OR 0.82 per year ( $95 \%$ CI $0.76-0.88$ ) and post-judgment trend OR 0.56 per year ( $95 \%$ CI 0.51-0.62); $\mathrm{p}<0.001$ for pre- vs post-judgment trend (Fig 3).

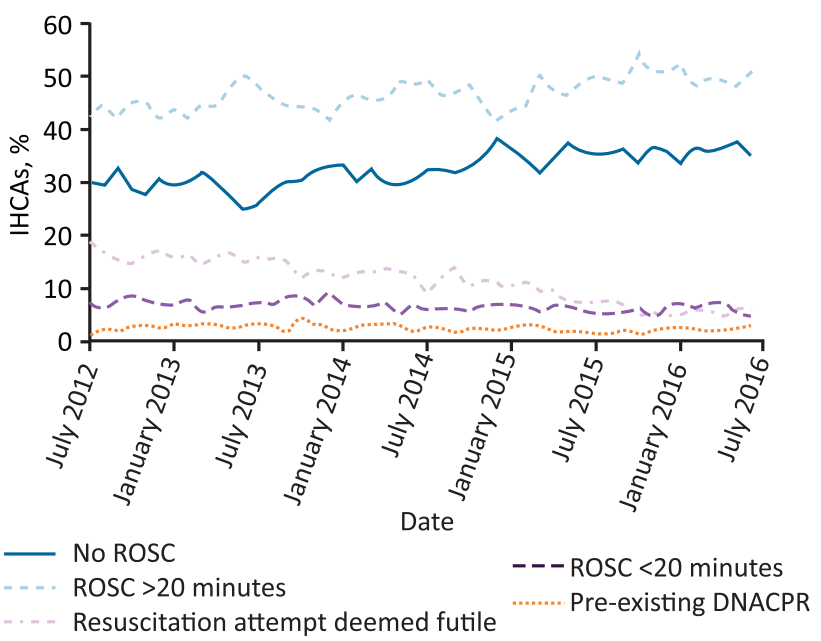

Fig 2. Plot of the proportion of IHCAs per month by reason resuscitation stopped. IHCAs = in-hospital cardiac arrests resulting in a resuscitation attempt; No ROSC = no return of spontaneous circulation; Pre-existing DNACPR = documented valid do not attempt cardiopulmonary resuscitation notice was discovered after cardiopulmonary resuscitation had commenced; Resuscitation attempt deemed futile $=$ resuscitation attempts were deemed futile after arrival of the resuscitation team; ROSC $<20$ minutes $=$ return of spontaneous circulation sustained for less than 20 minutes; ROSC $>20$ minutes $=$ return of spontaneous circulation sustained for more than 20 minutes.

The analyses of data for the two subgroups where IHCAs occurred in a ward and the patient had a hospital length of stay of $>24$ hours before the IHCA yielded similar results to the overall findings.

Following the judgment, there was no significant change in the proportion of medical admissions aged $>70$ years who remained on a ward rather than being admitted to an ICU following a successful resuscitation attempt (OR 1.20; $95 \%$ CI $0.71-2.03 ; p=0.49)$ and for change in trend (OR

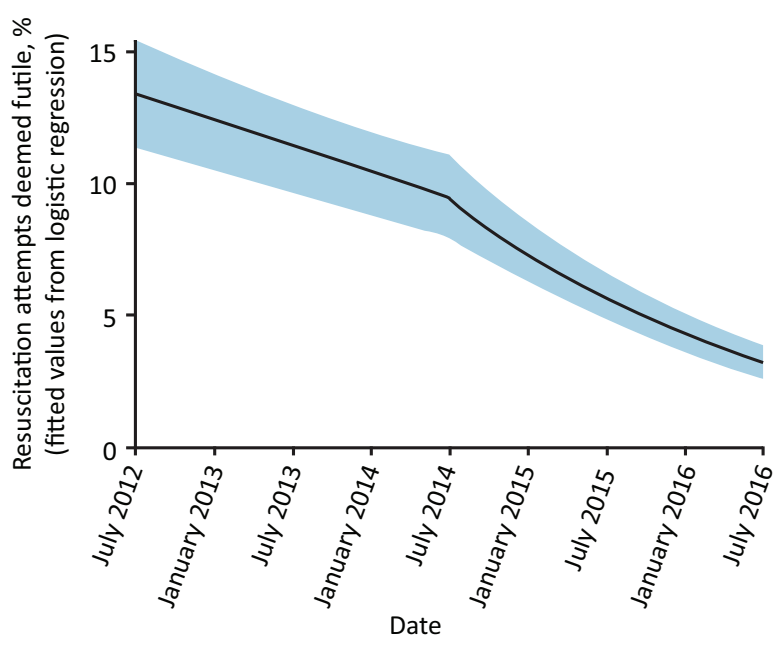

Fig 3. Interrupted time series analysis showing the impact of the Tracey judgment (published on 17 June 2014) on the proportion of resuscitation attempts deemed futile. 
1.00; $95 \%$ CI 0.98-1.02; $p=0.82$ ). However, the proportion of resuscitation attempts deemed futile within this subgroup increased significantly immediately following the judgment (OR 2.96; 95\% CI 1.98-4.43; $p<0.001$ ) and the downward trend present before the judgment continued at a steeper rate (pre-judgment trend OR 0.83 per year; $95 \%$ CI 0.75-0.93; post-judgment trend OR 0.51 per year; $95 \%$ CI 0.45-0.59; $\mathrm{p}<0.001$ for pre- vs post-judgment trend).

\section{Discussion}

In our study, the IHCA rate was decreasing before the judgment. However, there was an immediate increase in the rate following the judgment with a concurrent slowing in the rate of decline in the post-judgment period. The proportion of resuscitation attempts deemed futile was also decreasing before the judgment. In contrast to the IHCA rate, the rate of attempts deemed futile continued to decrease after the judgment. Compared with the pre-judgment period, the post-judgment rate of resuscitation attempts deemed futile fell faster. A possible surrogate measure for care limitation - the proportion of medical admissions aged $>70$ years who remained on a ward after an IHCA rather than being admitted to ICU following a successful resuscitation attempt - showed no change following the judgment.

Consequently, although the hypothesis that the judgment would result in an increase in IHCA rate was supported, the hypothesis that it would result in an increase in resuscitation attempts deemed futile was not.

This is the first study of the impact of the Tracey judgment on IHCA rates and resuscitation attempts deemed futile. Major strengths of the study include a large representative sample of UK hospitals; and clinical data collected to a high standard according to strict rules and definitions, validated locally and centrally, with few missing data (only four IHCAs excluded for this reason). One weakness of our study is that the remit of the NCAA is to audit the outcomes of the resuscitation team (or equivalent) and, therefore, the database includes only cardiac arrests involving a 2222 call. Patients having a cardiac arrest in a monitored area with specialist staff already present (eg the ICU) may well be resuscitated without a 2222 call having been made or the hospital-based resuscitation team attending, and we do not know the outcomes for these patients.

Although the interrupted time series analysis allows the analysis to take account of time trends and seasonality, there are assumptions and limitations to the statistical analysis. Time trends were assumed to be linear both before and after the judgment, and it is possible that mis-specification of the functional form may produce apparent effects where none exist. The absence of control hospitals that were not subject to any effects of the judgment means that we are unable to control for the impact of any other events coincident in time, leaving the potential for confounding. In addition, the analyses were not adjusted for patient factors as this would require patient-level data on all admissions to the study hospitals.

The immediate increase in the IHCA rate following the judgment, with the concurrent slowing in the rate of decline in the post-judgment period, could have several causes including a reduction in DNACPR decisions being made without patient/ family involvement, increased staff reluctance to discuss DNACPR options with patients, or an increase in patients choosing to have CPR. However, our data do not enable us to be certain of the actual cause.
Additionally, our study does not enable us to state reasons for the decrease in the proportion and rate of futile resuscitation attempts. Although futility is defined by NCAA, we do not know the precise reasons for deciding an attempt was futile. It is possible that that the Tracey judgment (or other factors around this time) led to continuation of resuscitation efforts, even in patients considered to have a poor outcome.

\section{Conclusion}

The study shows that the Tracey judgment was followed by an immediate increase in the rate of in-hospital cardiac arrests resulting in a resuscitation attempt while the proportion of resuscitation attempts deemed futile continued to decrease but at a greater rate than before. These changes may imply that some hospital staff avoided making DNACPR decisions, resulting in more IHCAs. However, the precise mechanism for these changes remains unclear.

\section{Supplementary material}

Additional supplementary material may be found in the online version of this article at www.rcpjournals.org/clinmedicine:

S1 - CONSORT flow diagram showing study exclusions.

S2 - Table showing descriptive statistics for the pre- and postjudgment periods.

\section{Funding}

This project was supported by internal funding from the Resuscitation Council (UK) and the Intensive Care National Audit \& Research Centre.

\section{Conflicts of interest}

Dr Reynolds, Prof Rowan, Dr Soar and Prof Smith are members of the NCAA Steering Group. Prof Nolan is editor-in-chief of Resuscitation and chair of the NCAA Steering Group.

\section{Acknowledgements}

The authors wish to thank the staff at hospitals participating in the National Cardiac Arrest Audit, the National Audit Programme Team at the Intensive Care National Audit \& Research Centre and the other members of the NCAA Steering Group.

\section{References}

$1 \quad R$ (Tracey) $v$ Cambridge University Hospitals NHS Foundation Trust \& Others: [2014] EWCA Civ 822. Royal Courts of Justice, 2014. www.judiciary.uk/wp-content/uploads/2014/06/traceyapproved.pdf [Accessed 11 March 2019].

2 Mockford C, Fritz Z, George R et al. Do not attempt cardiopulmonary resuscitation (DNACPR) orders: A systematic review of the barriers and facilitators of decision-making and implementation. Resuscitation 2015;88:99-113.

3 Nicolasora N, Pannala R, Mountantonakis S et al. If asked, hospitalized patients will choose whether to receive life-sustaining therapies. J Hosp Med 2006;1:161-7.

4 Gorton AJ, Jayanthi NVG, Lepping P, Scriven MW. Patients' attitudes towards 'do not attempt resuscitation' status. J Med Ethics 2008;34:624-6. 
5 Giles H, Moule P. Do not attempt resuscitation' decision-making: a study exploring the attitudes and experiences of nurses. Nurs Crit Care 2004;9:115-22.

6 Holland CL, Bowker LK, Myint PK. Barriers to involving older people in their resuscitation decisions: the primary-secondary care mismatch highlights the potential role of general practitioners. Int ] Clin Pract 2013;67:379-84.

7 Siddiqui MF, Holley JL. Residents' practices and perceptions about do not resuscitate orders and pronouncing death: an opportunity for clinical training. Am J Hosp Palliat Care 2011;28:94-7.

8 Deep KS, Griffith CH, Wilson JF. Discussing preferences for cardiopulmonary resuscitation: what do resident physicians and their hospitalized patients think was decided? Patient Educ Counsel 2008;72:20-5.

9 Deep KS, Green SF, Griffith CH, Wilson JF. Medical residents' perspectives on discussions of advanced directives: can prior experience affect how they approach patients? J Palliat Med 2007;10:712-20.

10 Sulmasy D, Sood JR, Texiera K et al. A prospective trial of a new policy eliminating signed consent for do not resuscitate orders. J Gen Intern Med 2006;21:1261-8.
11 Myint PK, Miles S, Halliday DA, Bowker LK. Experiences and views of specialist registrars in geriatric medicine on 'do not attempt resuscitation' decisions: a sea of uncertainty? QJM 2006:99:691-700

12 Nolan JP, Soar J, Smith GB et al. Incidence and outcome of in-hospital cardiac arrest in the United Kingdom National Cardiac Arrest Audit. Resuscitation 2014;85:987-92.

13 Bernal JL, Cummins S, Gasparrini A. Interrupted time series regression for the evaluation of public health interventions: a tutorial. Int J Epidemiol 2017;46:348-55.

14 Harrison DA, Patel K, Nixon E et al. Development and validation of risk models to predict outcomes following in-hospital cardiac arrest attended by a hospital-based resuscitation team. Resuscitation 2014:85:993-1000.

Address for correspondence: Prof Gary B Smith, Centre of Postgraduate Medical Research and Education (CoPMRE), Faculty of Health and Social Sciences, Bournemouth University, Bournemouth BH1 3LT, UK. Email: gbsresearch@virginmedia.com

\section{Outpatients: the future - adding value through sustainability}

Published in 2018, this report from the Royal College of Physicians argues for

new models of outpatient care.

\section{Download the report and}

supplementary case studies at

www.rcplondon.ac.uk/outpatients-future-sustainability 